\title{
HETEROGENEITY OF hCG IN COMMERCIAL PREPARATIONS OF HUMAN CHORIONIC GONADOTROPIN (hCG) AND HUMAN MENOPAUSAL GONADOTROPIN (hMG)
}

\author{
Laura Riccetti $^{1}$, Elisa Pignatti ${ }^{1,2}$, Manuela Simoni ${ }^{1,3}$
}

${ }^{1}$ Unit of Endocrinology, Department of Biomedicine, Metabolism and Neural Sciences, University of Modena and Reggio Emilia, Modena, Italy 2 Center for Genomic Research, University of Modena and Reggio Emilia, Modena, Italy ${ }^{3}$ Department of Medicine, Endocrinology, Metabolism and Geriatrics, Azienda AUSL of Modena, NOCSAE, Modena, Italy

\section{INTRODUCTION}

Human chorionic gonadotropin (hCG) is a glycoprotein hormone composed by two subunits, an alpha subunit common to all gonadotropins and a specific beta subunit ${ }^{1}$. hCG is produced by trophoblast cells as differently glycosylated isoforms with a wide range of molecular weights, from 30 to $50 \mathrm{KDa}$ ("regular" $\mathrm{hCG}$ is $37 \mathrm{KDa}$ ) ${ }^{2}$. hCG is used in the treatment of infertility ${ }^{3}$.

\section{DESIGN}

Three hCG preparations from pregnancy urine (50mIU Gonasi-IBSA, 50mIU Pregnyl-Organon, 70mIU Sigma C0434), one recombinant (5ng r$\mathrm{hCG}$, Ovidrel-Serono) and an urinary hMG (75mIU Meropur-Ferring) were compared by $12 \%$ SDS-PAGE and Western Blotting using a polyclonal antibody against hCG beta subunit (Dako A0231). Different conditions were used in processing all the preparations such as denaturing and reducing samples or preserving their "native" conformation. The Western blotting patterns were confirmed by Silver Staining and ConA Affinity Chromatography.

\section{OBJECTIVE}

The aim of this study is to analyze and compare the composition of different commercial hCG preparations

\section{RESULTS}

Despite no particular differences were observed under denaturing-reducing conditions, we found qualitatively and quantitatively peculiar patterns under "native" conditions. In the urinary hCG preparations up to three isoforms were found with different molecular weights $(32,37$ and $45 \mathrm{KDa}$; $\mathrm{n}=4$ ). In contrast, in $\mathrm{r}-\mathrm{hCG}$ four variants were found (one doublet at 31$32 \mathrm{KDa}$ and a doublet more expressed at 44-45 KDa; $\mathrm{n}=4$ ) (Fig. 1). All preparations, including $\mathrm{hMG}$, seem to contain hyperglycosylated hCG (hCG-H) of about $45 \mathrm{KDa}$ which was characterized against hCG-H purified from human choriocarcinoma cell line (JEG-3) culture medium as control. Silver Staining (Fig. 2) shows all the proteins contained in the samples while ConA chromatography (Fig. 3) confirming that all the isoforms detected were glycosylation variants of hCG.

\section{CONCLUSIONS}

The commercial hCG and hMG preparations consist of several isoforms which differ in molecular weight and grade of glycosylation. Surprisingly, r-hCG has a wider range of glycosylated variants than urinary hCG. The peculiar signal pattern observed by Western Blotting demonstrates the heterogeneity of hCG in commercial preparations.

\section{REFERENCES}

Lapthorn AJ, Harris DC, Littlejohn A, Lustbader JW, Canfield RE, Machin KJ (1994) Crystal structure of hCG. Nature 369:455-61.

2. Cole LA (2012) hCG, five independent molecules. Clin Chim Acta 413(1-2):48-65.

3. Lunenfeld (2002) Development of gonadotrophins for clinical use. Reprod Biomed Online 4 Suppl 1:11-7

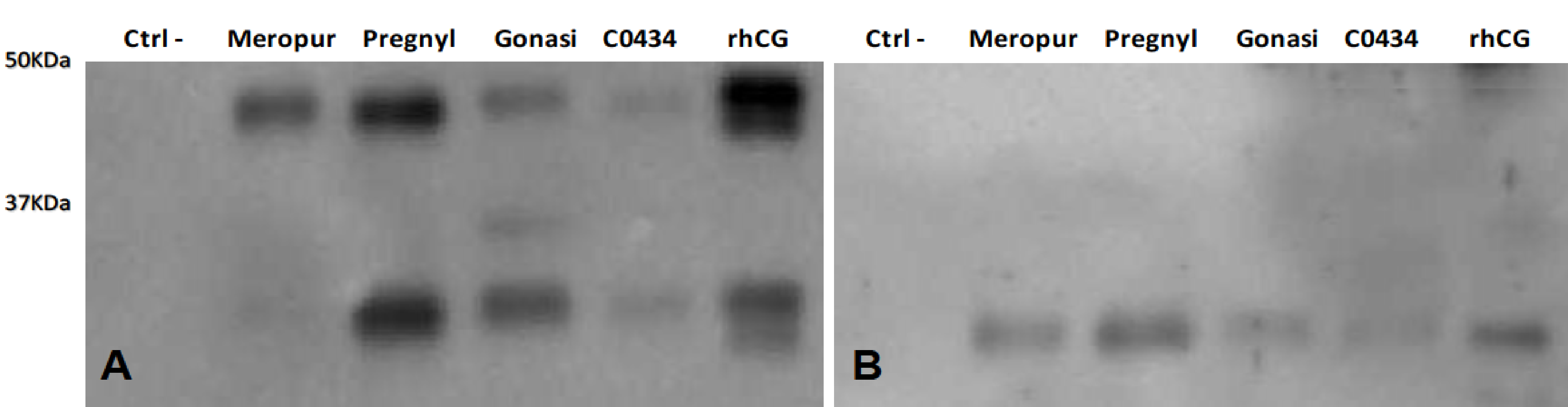

Figure 1. 12\% SDS-PAGE and Western Blotting of hCG and hMG preparations under different conditions. (A) Samples under native conditions; (B) Samples under denaturing and reducing conditions. From the left: negative control, $75 \mathrm{mIU}$ Meropur, 50mIU Pregnyl, 50mIU Gonasi, 70mIU C0434 Sigma, 5ng rhCG Ovidrel; (n=4).

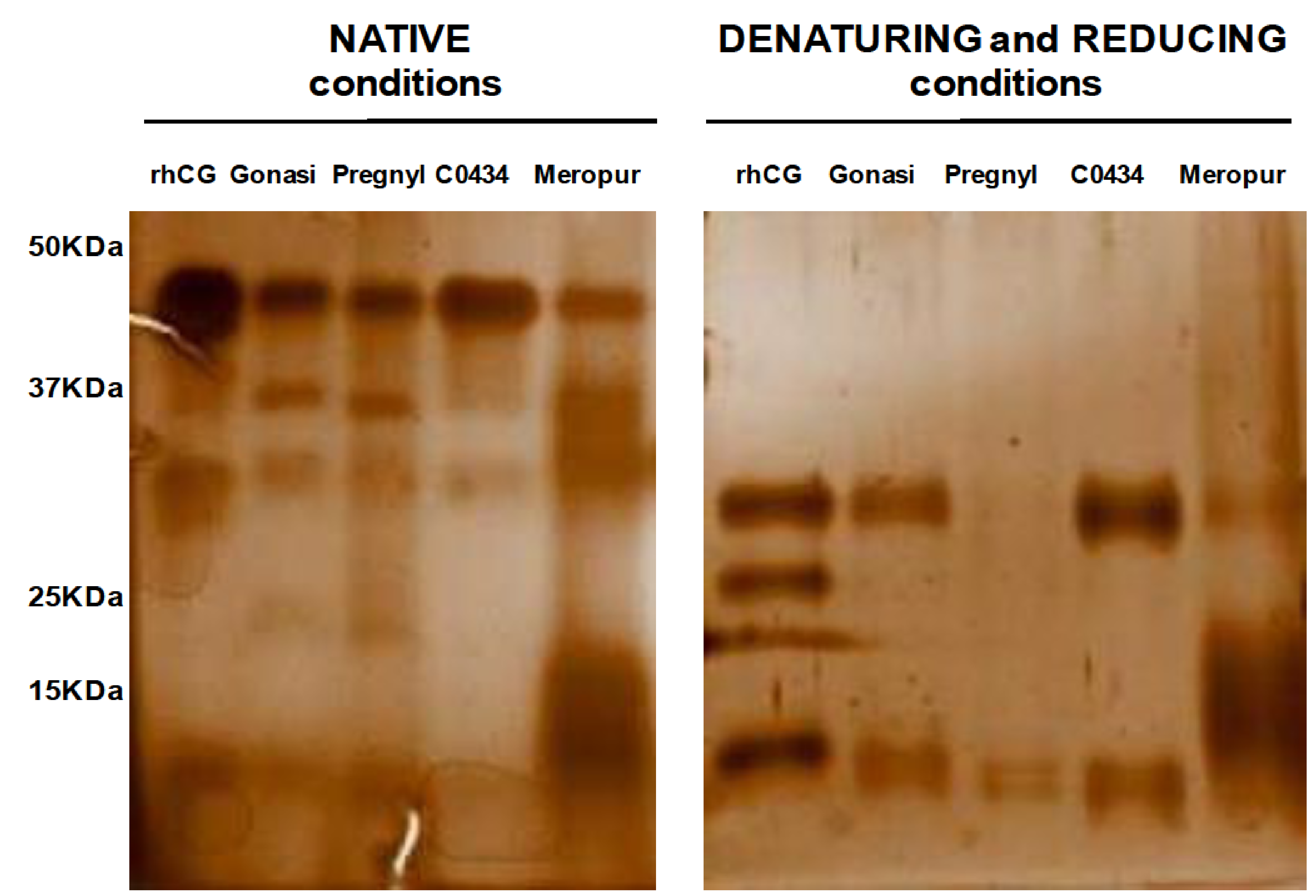

Figure 2. Silver Staining of the different hCG preparations under native and denaturing-reducing conditions. From the left: $150 \mathrm{ng}$ rhCG Ovidrel, 1,5 IU Gonasi, 1,5 IU Pregnyl, 3 IU Sigma C0434, 2 IU Meropur.
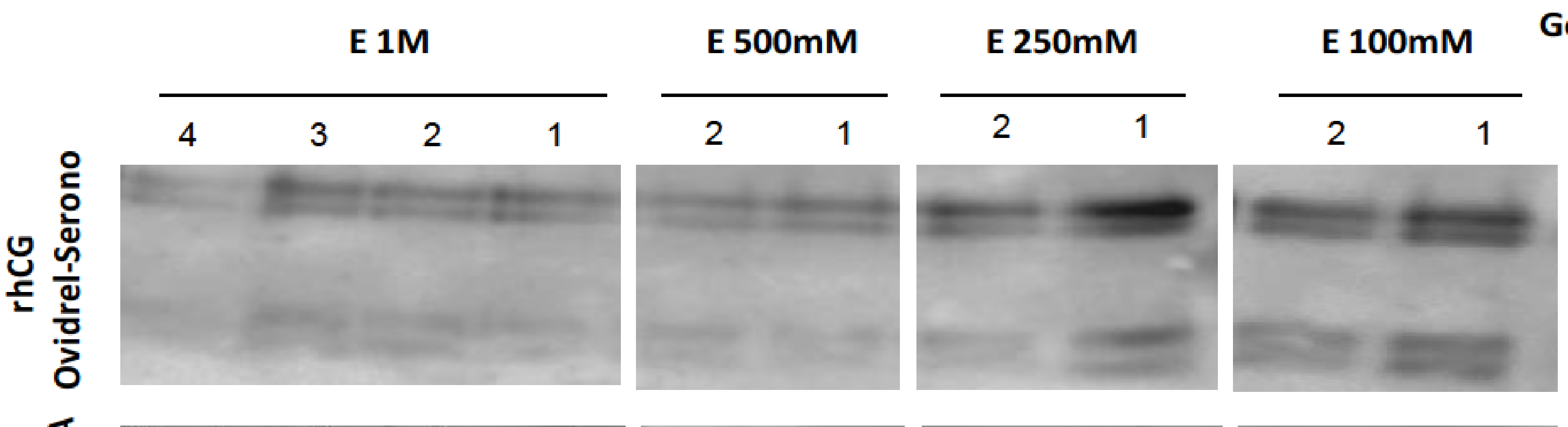

loaded
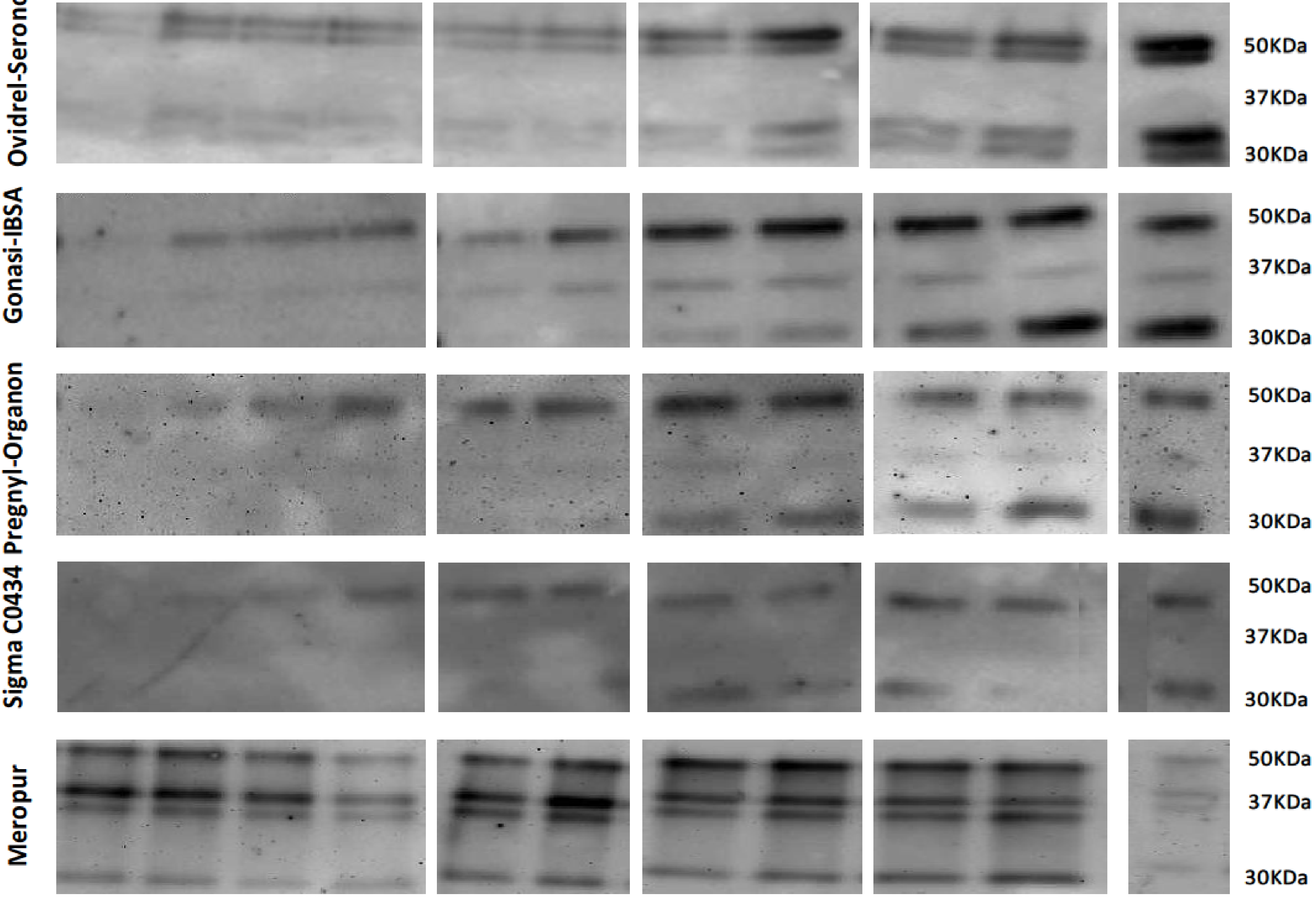

Figure 3. Western Blotting of the eluted fractions by ConA chromatography comparing different hCG preparations. $1 \mu \mathrm{g}$ rhCG Ovidrel, 10IU Gonasi, 10IU Pregnyl, 2IUSigma C0434, 10IU Meropur were loaded onto a ConA column and a step gradient elution $(100 \mathrm{mM}, 250 \mathrm{mM}, 500 \mathrm{mM}$ and $1 \mathrm{M}$ of $\alpha$-D-methylglucopyranoside) was applied with a $0.5 \mathrm{ml} / \mathrm{min}$ flow rate. A total of $10 \mathrm{ml}$ of elution fractions were recovered.

\section{ASEAM | ASSOCIAZIONE SCIENTIFICA Winner of ESE Basic Science Meeting Grant

